\title{
BOTULINUM TOXIN TYPE-A IN THE MANAGEMENT OF CEREBRAL PALSY: LOW OR HIGH DOSE?
}

\author{
Alper I. Dai ${ }^{1}$ and Mohammad Wasay ${ }^{2}$ \\ 1D epartment of Pediatric N eurology, School of Medicine, U niversity of G aziantep, Gaziantep, Turkey; and 2 Section of \\ Neurology, Aga Khan University, Karachi, Pakistan \\ Corresspondence to: Dr. Mohammad Wasay, Associate Professor, Department of Medicince (Neurology), Aga Khan University Hospital, Karachi 74800, Pakistan.
}

Tel: (92-21) 493-0051 Ext. 4665, 4681; fax: (92-21) 493-4294; Email: mohammad.wasay@ aku.edu

\begin{abstract}
Cerebral palsy is the most common cause of severe physical disability in childhood. Spasticity is a common and disabling symptom for many patients with cerebral palsy. Therapy for spasticity is symptomatic with the aim of increasing functional capacity and relieving discomfort. Spasticity treatment by orally administered drugs and intramuscular chemodenervation agents has become more frequent. Most oral medications to treat spasticity have been inadequately studied in children, especially those with cerebral palsy. Since its first use in pediatric patients, reported in 1993, botulinum toxin (BTX-A), a relatively recent addition to the available medical interventions for children with cerebral palsy, has rapidly gained acceptance as a treatment of spasticity. The clinical effects of BTX-A have been reported to include decreased spasticity and increased range of motion. However, no consensus exists among clinicians about how an optimal dose of BTX-A should be determined and there are no standard guidelines on doses of BTX-A in children. Doses of 2-6 U/kg with a maximum total dose of $29 \mathrm{U} / \mathrm{kg}$ have been reported. Although there are no standard guidelines on doses in children. The current practice is to inject BTX-A at higher doses than reported in the past. Larger dose of BTX-A is used more frequently, which is considered safe, more effective, and better tolerated by children. Titration of the dose of BTX-A is necessary because muscle spasticity affects different patients in different ways. The dosage of BTX-A must be individualized for each patient.
\end{abstract}

Cerebral palsy is a group of persistent movements or postural disturbances secondary to a nonprogressive lesion in a developing brain, and is considered the main cause of motor disability in children, affecting 1 in 5001000 newborns. It is the most common cause of severe physical disability in childhood. ${ }^{1}$ Among patients with cerebral palsy, $70 \%$ have spasticity, which is responsible for hamstring flexion, hip adduction, and equinus foot, resulting in variable functional disabilities. More than $50 \%$ of patients with the disorder can walk without arm assistance, $25 \%$ cannot walk, and $30 \%$ are mentally impaired. ${ }^{2}$

Spasticity is a common and disabling symptom for many patients with upper motor neuron dysfunction. It results from interruption of inhibitory descending spinal motor pathways. Although the pathophysiology of spasticity is poorly understood ${ }^{3}$, the final common pathway is overactivity of the alpha motor neuron. Muscle overactivity, one of the cardinal features of spasticity, is a common sequel of cerebral palsy. Spasticity is responsible for several limitations that interfere with gait, causing variable functional disability. ${ }^{4}$

Therapy for spasticity is symptomatic with the aim of increasing functional capacity and relieving discomfort. Any approach to treatment should be multidisciplinary, including physical therapy, and possibly surgery, as well as pharmacotherapy. Since 1980, modification of spasticity by orally administered drugs, intramuscular chemodenervation agents, intrathecally administered drugs and surgery has become more frequent. 5

A variety of oral medications have been used to diminish the sensitivity of local nerves and muscles to control their reactions to environmental stimuli that result in muscle spasticity or involuntary movements. These medications are often only of modest benefit because of undesirable side effects ${ }^{6}$. There are newer oral agents recognized for their potential antispasticity properties. They include 
tizanidine, clonazepam and clonidine. Tizanidine has been studied extensively in adults with spasticity from a variety of causes and has been found to be of proven benefit, but not very much is known yet of its role for childhood cerebral palsy. Most oral medications to treat spasticity have been inadequately studied in children, especially those with cerebral palsy. 5,7

For the walking child with spastic cerebral palsy, the objective is to counter or minimise the negative long term effect of spasticity and weakness during the period of growth. Simple measures of stretching, walking, and orthotic use can be supplemented by oral muscle relaxants and particularly botulinum toxin typeA. The use of intramuscular botulinum toxin to block neuromuscular transmission in spastic muscles has become more popular since its initial use with pediatric patients, reported in 1993.8 Its prime use has been in calf muscles to overcome dynamic equinus of the ankle in children with hemiplegia or diplegia. The effects of botulinum toxin are transitory and injections must be repeated every 4 to 6 months. ${ }^{9}$

With the advent of botulinum toxin type A (BTX-A) as an adjuvant treatment of patients with equinus foot, several studies have been reported demonstrating that BTX-A can be one of the main tools to improve prognosis in children with cerebral palsy. Botulinum toxin, a relatively recent addition to the available medical interventions for children with cerebral palsy, has rapidly gained acceptance as a treatment that temporarily reduces focal muscle spasticity 10 . The clinical effects of BTX-A have been reported to include decreased spasticity and increased range of motion. These effects may be critically important when considering the influence of spasticity, limited muscle length, and restricted range of motion on the growing bones of young children. Controlled studies, demonstrating the efficacy of BTX-A in treatment of childhood cerebral palsy have led to approval for this indication in many European countries ${ }^{9}$. The greatest pitfalls are created by the lack of established protocols for the walking child with spastic cerebral palsy. ${ }^{11,12}$

Doses of 2-6 U/kg body weight per muscle with a maximum total dose of $29 \mathrm{U} / \mathrm{kg}$ have been reported. Although there are no standard guidelines on doses in children, a review of previous publications indicates that the dose that has been used for children with cerebral palsy has increased over time. The current practice is to inject several muscles at each injection session, with smaller patients receiving higher doses than reported in the past. 8,13

The dosage of BTX-A must be individualized for each patient. A recent consensus statement recommended a dose of 12 units of Botox (Allergan, USA) per kg bodyweight, with a maximum of 300 units Botox per child on one occasion, and 50 units per injection site. ${ }^{4}$ The recommended treatment interval is $6-12$ months, with a minimum of 3 months. It has become apparent that larger doses used less frequently are safe, more effective, better tolerated by children, more acceptable to their families, and the functional outcomes are longer lasting. ${ }^{14}$ Dosages of 16-24 units per $\mathrm{kg}$ bodyweight are now in use in experienced hands for double or triple level lower limb sites. However, the dose-efficiency relationship might only be linear for a distinct range. Clinicians should be aware of unwanted effects during the application of the drug. $11,15,16$

The mean dose of BTX-A used varies between treatment centres. This cannot be entirely explained on the basis of different patient populations. The use of a small dose of BTX-A may reflect a cautious attitude of the practitioner or an attempt to reduce the cost of treatment. An alternative explanation is that BTX-A is used in conjunction with other antispasticity treatments. Similarly, the variability between examiners in the evaluation of the clinical effectiveness of BTX-A may be a factor. 17,18

The clinician may inject a large dose of BTX-A to achieve a desired reduction in muscle tone in a given patient but may use a smaller dose in another subject, although both patients have the same degree of muscle spasticity. This need to titrate the dose of BTX-A is necessary because muscle spasticity affects different patients in different ways and the treatment decision in each case is usually based upon therapeutic goals. The choice of therapeutic interventions is also influenced by locally available expertise and resources. 19

Three randomised, double-blind dose-ranging studies have examined the effects of various doses of BTX-A on gait measures in patients with cerebral palsy. In these studies, the higher doses of the preperations were more effective in improving gait measures than the lower doses.1,20

What is the dose of BTX-A in cerebral palsy? There is some debate concerning optimum treatment of BTD-A in the non-ambulatory child. The greatest pitfalls are created by a lack of established protocols for the walking child with spastic cerebral palsy. There is currently no consensus among clinicians about how an optimal dose of BTX-A should be determined, and there are no standard guidelines on doses of BTX-A in children. ${ }^{1}$

There is little doubt that BTX-A has been an outstanding advance in recent years, creating important opportunities to enable our therapy and treatments to be significantly more effective in the management of cerebral palsy. More 
controlled trials are now needed to confirm the antispasticity effects of BTX-A with larger patients groups for more accurate results.

\section{REFERENCES}

1. Koman LA, Smith BP, Shilt JS. Cerebral palsy. Lancet 2004; 363:1619-1631.

2. Tilton $\mathrm{AH}$. Management of spasticity in children with cerebral palsy. Semin Pediatr Neurol March 2004; 58-65.

3. Patel DR, Soyode O. Pharmacologic interventions for reducing spasticity in cerebral palsy. Ind J Ped 2005; 72:869-872.

4. Flett PJ. Rehabilitation of spasticity and related problems in childhood cerebral palsy. J Paediatr Child Health 2003; 39(1):6-14.

5. Al-Shahrani AM. Anti-spasticity medications. Saudi Med J 2003; 24(1):19-22.

6. Gormley ME. Treatment of neuromuscular and musculoskeletal problems in cerebral palsy. Pediatr. Rehabil 2001; 4: 516.

7. Goldstein M, Harper DC. Management of cerebral palsy: equinus gait. Dev. Med. Child Neurol 2001; 43:563 9.

8. Graham HK, Aoki KR, Autti-Ramo I, et al. Recommendations for the use of botulinum toxin type $A$ in the management of cerebral palsy. Gait Posture 2000; 11:67-79.

9. Jefferson RJ. Botulinum toxin in the management of cerebral palsy. Dev Med Child Neurol 2004; 46:491-499.

10. Koman LA, Mooney JF, Smith BP, et al. Botulinum toxin type A neuromuscular blockade in the treatment of lower extremity spasticity in cerebral palsy: a randomized, double-blind, placebocontrolled trial. J Pediatr Orthop 2000; 20:108115.

11. Edgar TS. Clinical utility of botulinum toxin in the treatment of cerebral palsy: comprehensive review. J Child Neurol 2001; 16:37-46.

12. Kita M, Goodkin DE. Drugs used to treat spasticity. Drugs 2000; 59:487-495.

13. Pidcock F.S. The emerging role of therapeutic botulinum toxin in the treatment of cerebral palsy, J Pediatr 2004; 145:S33-S35.

14. Wissel J, Heinen F, Schenkel A, et al. Botulinum toxin $A$ in the management of spastic gait disorders in children and young adults with cerebral palsy: a randomized, double-blind study of "high-dose" versus "low-dose" treatment. Neuropediatrics 1999; 30:120-124.

15. Ubhi T, Bhakta BB, Ives HL, et al. Randomised double blind placebo controlled trial of the effect of botulinum toxin on walking in cerebral palsy. Arch
Dis Child 2000; 83:481-487.

16. Ade-Hall RA, Moore AP. Botulinum toxin type A in the treatment of lower limb spasticity in cerebral palsy. Cochrane Database Syst Rev 2000; (2):1408.

17. Priess RA, Condie DN, Rowley DI, Graham HK. The effects of botulinum toxin (Btx-A) on spasticity of the lower limb and on gait in cerebral palsy. J Bone J oint Surg Br 2003; 85:943-948.

18. Houltram J, Noble I, Boyd RN, Corry I, Flett P, Graham HK. $\neg$ Botulinum toxin type A in the management of equinus in children with cerebral palsy: an evidence-based economic evaluation. Euro J Neurol 2001; 8(Suppl. 5):194_202.

19. Wong V. Use of botulinum toxin injection in 17 children with spastic cerebral palsy. Pediatr Neurol 1998; 18:124-130.

20. Baker R., Jasinski and MaciagTymecka l.et al. Botulinum toxin treatment of spasticity in diplegic cerebral palsy A randomized, double-blind, placebocontrolled, dose-ranging study. Dev Med Child Neurol 2002; 44:666-675. 